\title{
Degenerate fold-Hopf bifurcations in a Rössler-type system
}

\author{
G. TIGAN \\ Department of Mathematics, \\ Politehnica University of Timisoara, Romania \\ gheorghe.tigan@upt.ro \\ J. LLIBRE \\ Departament de Matemàtiques, \\ Universitat Autònoma de Barcelona, Catalonia, Spain \\ jlibre@mat.uab.cat \\ L. CIURDARIU \\ Department of Mathematics, \\ Politehnica University of Timisoara, Romania \\ loredana.ciurdariu@upt.ro
}

Received (to be inserted by publisher)

We study the Hopf and the fold-Hopf bifurcations of the Rössler-type differential system

$$
\dot{x}=-y-z, \quad \dot{y}=x+a y, \quad \dot{z}=-c z+b y z,
$$

with $b \neq 0$. We show that the classical Hopf bifurcation cannot be applied to this system for detecting the fold-Hopf bifurcation, which here is studied using the averaging theory. Our results show that a Hopf bifurcation takes place at the equilibrium $(-a c / b, c / b,-c / b)$ when $c=a<0$. This Hopf bifurcation becomes a fold-Hopf bifurcation when $c=a=0$.

Keywords: Rössler system, periodic orbits, averaging theory, zero-Hopf bifurcation, fold-Hopf bifurcation.

\section{Introduction}

Fold-Hopf bifurcation occurs in minimum three-dimensional systems having minimum two independent parameters. To define it, we consider a differential system in the form

$$
\dot{x}=f(x, \alpha),
$$

$x \in \mathbb{R}^{3}, \alpha=\left(\alpha_{1}, \alpha_{2}\right) \in \mathbb{R}^{2}, f$ smooth, which has an equilibrium $x=O(0,0,0)$ for all $\|\alpha\|=\sqrt{\alpha_{1}^{2}+\alpha_{2}^{2}}$ small enough. Assume that for $\left(\alpha_{1}, \alpha_{2}\right)=(0,0)$, the Jacobian matrix $J_{0}$ of the system (1) at $O$ has two purely imaginary eigenvalues $\pm i \omega_{0}$ and one real eigenvalue equal to 0 , for some $\omega_{0}>0$. This is known in the literature as the fold-Hopf (or zero-Hopf) bifurcation. Since the eigenvalues of $J_{0}$ are assumed simple, the eigenvalues of the Jacobian matrix $J_{\alpha}$ of the system (1) at $O$ for $\|\alpha\|$ small enough are of the form $\lambda_{1}=\nu(\alpha), \lambda=\mu(\alpha)+i \omega(\alpha)$ and $\bar{\lambda}$, such that $\nu(0)=\mu(0)=0$ and $\omega(0)=\omega_{0}>0$.

The study of this bifurcation, that is, the study of the behavior of the system (1) when $\|\alpha\|$ is small enough, is not trivial. In order to study it, the author of [Kuznetsov, 1995] imposed five other conditions, 
G1-G5, to be able to describe some generic properties of the system (1) when $\|\alpha\|$ is small enough. He used an approach based on the normal forms theory (see also [Algaba et al., 1998]). We explained briefly in the paper's Appendix how the G1-G5 generic conditions arise. When the conditions are satisfied we say the fold-Hopf bifurcation is non-degenerate, otherwise, degenerate. The bifurcation was well studied in the non-degenerate context in [Kuznetsov, 1995]. All five generic conditions are crucial for the results obtained in this case. A natural question arises: what happens when one or more of the G1-G5 conditions are broken, that is, when the fold-Hopf bifurcation becomes degenerate? The motivation of the present work is to try to bring a partial answer to this question by studying the existence of periodic orbits in a particular three-dimensional system that has a degenerate fold-Hopf bifurcation. Moreover, because this kind of bifurcation appears in many differential systems in $\mathbb{R}^{3}$, we illustrate how it can be studied using the averaging theory. More exactly, we introduce in this paper a Rössler-type system of the form

$$
\dot{x}=-y-z, \quad \dot{y}=x+a y, \quad \dot{z}=-c z+b y z,
$$

where $a, b, c$ are real parameters and $b \neq 0$.

We say that the system (2) is of Rössler-type because it has a single nonlinear term and the first two equations of the system (2) are identical to the Rössler system [Rössler, 1976], which is well-known in the literature and has been used successfully in applications (secure communications, synchronizations, etc.) because it has one of the simplest analytical forms but displays complex dynamics, including chaos. We assume here $b \neq 0$, otherwise the differential system (2) becomes linear, and consequently it would have a trivial dynamics.

As we shall see in section 2 the classical theory for studying the fold-Hopf bifurcation does not work for the Rössler-type differential system (2), but we shall study it using the averaging theory for studying the bifurcations of periodic solutions from a zero-Hopf bifurcation. This tool for studying the zero-Hopf bifurcation is very useful and can be adapted to many different kind of differential equations, see for instance [Castellanos et al., 2013; Euzébio et al., 2015; Llibre et al., 2014; Wei et al., 2016].

\section{Degeneracy of the fold-Hopf bifurcation}

The equilibria of $(2)$ are $O(0,0,0)$ and $A(-a c / b, c / b,-c / b)$ because $b \neq 0$. The points $O$ and $A$ collide when the parameter $c=0$ and the coinciding equilibria have the eigenvalues 0 and $a / 2 \pm i \omega$, where $\omega=\sqrt{4-a^{2}} / 2$ whenever $-2<a<2$. It follows that the system (2) may undergo a fold-Hopf bifurcation at $a=0$, when the eigenvalues are $0, \pm i$.

Denote further by $\alpha=(a, c)$. Writing (2) in form (15) (see section 5), one can compute effectively the eigenvectors of the Jacobian matrix $J(\alpha)$ of system (2) at $O$ for all $\alpha$. Indeed, the eigenvalues of $J(\alpha)$ are $\lambda_{1}=-c$, respectively $\lambda_{ \pm}=a / 2 \pm i \omega$, with the corresponding eigenvectors

$$
q_{0}=\left(\begin{array}{c}
\frac{a+c}{c^{2}+a c+1} \\
-\frac{1}{c^{2}+a c+1} \\
1
\end{array}\right), \quad q_{ \pm}=\left(\begin{array}{c}
-\frac{1}{2} a \pm i \omega \\
1 \\
0
\end{array}\right) .
$$

The adjoint eigenvectors $p_{0}(\alpha)$ and $p_{ \pm}(\alpha)$ are

$$
p_{0}=\left(\begin{array}{l}
0 \\
0 \\
1
\end{array}\right), \quad p_{ \pm}=-\frac{1}{4 \omega^{2} \mp 2 i a \omega}\left(\begin{array}{c}
-a \mp 2 i \omega \\
-2 \\
2 \frac{a \pm 2 i \omega}{a+2 c \mp 2 i \omega}
\end{array}\right) .
$$

Using the transformation $u=\left\langle p_{0}(\alpha), X\right\rangle, v=\left\langle p_{1}(\alpha), X\right\rangle$, where $X=(x y z)^{T}$, the system 
becomes

$$
\begin{aligned}
\dot{u} & =-c u-\frac{b}{1+a c+c^{2}} u^{2}+b u v+b u \bar{v}, \\
\dot{v} & =\left(\frac{1}{2} a+i \omega\right) v+\frac{b}{4} \frac{2 \omega+(a+2 c) i}{\omega\left(1+a c+c^{2}\right)}\left(-\frac{1}{1+a c+c^{2}} u^{2}+u v+u \bar{v}\right) .
\end{aligned}
$$

At $\alpha=(0,0)$ we have $B(0)=-b \neq 0, D(0)=b / 2$ and $G_{011}(0)=0$. The expressions of these coefficients are given in section 5 . Hence, the fold-Hopf bifurcation of system (2) is degenerate with respect to (G.2) $G_{011}(0) \neq 0$. Therefore, system (2) cannot be put in the normal forms (19), (20) or (21). Yet system (2) can be brought to the Poincaré normal form (18), because this uses only the first generic condition, (G.1) $g_{200}(0)=-b \neq 0$, which is still valid. However, the Poincaré form does not offer significant tools in studying the system. The implications on the system's dynamics of this degeneracy cannot be inferred from what is known presently in the literature, what makes the system worth studying as a case study for this degeneracy. But we can use the averaging theory as it was done in the paper [Llibre, 2014] for a partial study of this degenerate fold-Hopf bifurcation.

The next result provides a first order approximation for the periodic solutions of a non-autonomous periodic differential system using the averaging theory for computing periodic solutions, for a proof see Theorems 11.5 and 11.6 of [Verhulst, 1991].

Consider the differential system

$$
\dot{\mathbf{x}}=\varepsilon F(t, \mathbf{x})+\varepsilon^{2} G(t, \mathbf{x}, \varepsilon),
$$

with $\mathbf{x} \in D$, where $D$ is an open subset of $\mathbf{R}^{n}, t \geq 0$. We suppose that both $F(t, \mathbf{x})$ and $G(t, \mathbf{x}, \varepsilon)$ are $T$-periodic in $t$. We define the averaged function

$$
f(\mathbf{x})=\frac{1}{T} \int_{0}^{T} F(t, \mathbf{x}) d t .
$$

Theorem 1. Assume that

(i) $F$, its Jacobian $\partial F / \partial x$, its Hessian $\partial^{2} F / \partial x^{2}, G$ and its Jacobian $\partial G / \partial x$ are defined, continuous and bounded by a constant independent of $\varepsilon$ in $[0, \infty) \times D$ and $\varepsilon \in\left(0, \varepsilon_{0}\right]$.

(ii) $F$ and $G$ are $T$-periodic in $t$ ( $T$ independent of $\varepsilon$ ).

Then the following statements hold.

(a) If $p$ is a zero of the averaged function $f(\mathbf{x})$ and

$$
\left.\operatorname{det}\left(\frac{\partial f}{\partial \mathbf{x}}\right)\right|_{\mathbf{x}=p} \neq 0
$$

then there exists a $T$-periodic solution $\mathbf{x}(t, \varepsilon)$ of equation (4) such that $\mathbf{x}(0, \varepsilon) \rightarrow p$ as $\varepsilon \rightarrow 0$.

(b) If all the eigenvalues of the Jacobian matrix ( $\partial f / \partial \mathbf{x})$ have negative real part, then the periodic solution $\mathbf{x}(t, \varepsilon)$ is asymptotically stable. If some of these eigenvalues have positive real parts, this periodic orbit is unstable.

Our first main result is the following.

Theorem 2. Let $(a, c)=(\varepsilon \alpha, \varepsilon \gamma), b \neq 0$ and $\varepsilon$ a sufficiently small parameter. If $(\alpha-\gamma) \gamma \neq 0$, then the Rössler-type system (2) has a fold-Hopf bifurcation at the equilibrium point localized at the origin of coordinates when $\varepsilon=0$, and a periodic orbit $(x(t, \varepsilon), y(t, \varepsilon), z(t, \varepsilon))$ borns at this equilibrium for $\varepsilon>0$ sufficiently small satisfying

$$
(x(0, \varepsilon), y(0, \varepsilon), z(0, \varepsilon))=\left(0, \frac{\varepsilon \gamma}{b}, \frac{\varepsilon \gamma}{b}\right)+O\left(\varepsilon^{2}\right) .
$$

Moreover, this periodic solution is asymptotically stable if $\alpha-\gamma<0$ and $\gamma<0$, otherwise it is unstable. 
Proof. If $(a, b, c)=(\varepsilon \alpha, b, \varepsilon \gamma)$ with $\varepsilon>0$ a sufficiently small parameter, then the Rössler-type system becomes

$$
\begin{aligned}
& \dot{x}=-y-z, \\
& \dot{y}=x+\varepsilon \alpha y, \\
& \dot{z}=-\varepsilon \gamma z+b y z .
\end{aligned}
$$

Doing the rescaling of the variables $(x, y, z)=(\varepsilon X, \varepsilon Y, \varepsilon Z)$, system $(7)$ in the new variables $(X, Y, Z)$ writes

$$
\begin{aligned}
& \dot{X}=-Y-Z, \\
& \dot{Y}=X+\varepsilon \alpha Y, \\
& \dot{Z}=-\varepsilon(\gamma Z+b Y Z) .
\end{aligned}
$$

Now we shall write the linear part at the origin of the differential system (8) when $\varepsilon=0$ into its real Jordan normal form, i.e. as

$$
\left(\begin{array}{ccc}
0 & -1 & 0 \\
1 & 0 & 0 \\
0 & 0 & 0
\end{array}\right) .
$$

For doing that we consider the linear change $(X, Y, Z) \rightarrow(u, v, w)$ of variables given by $X=v, Y=-u-w$, $Z=-w$. In these new variables $(u, v, w)$ the differential system $(8)$ writes

$$
\begin{aligned}
\dot{u} & =-v+\varepsilon\left(\alpha u+(\alpha+\gamma) w+b u w+b w^{2}\right), \\
\dot{v} & =u, \\
\dot{w} & =-\varepsilon(\gamma+b u+b w) w .
\end{aligned}
$$

Now we pass the differential system (9) to cylindrical coordinates $(r, \theta, w)$ defined by $u=r \cos \theta$ and $v=r \sin \theta$, and we obtain

$$
\begin{aligned}
\dot{r} & =\varepsilon \cos \theta\left(\alpha \cos \theta r+(\alpha+\gamma) w+b \cos \theta r w+b w^{2}\right), \\
\dot{\theta} & =1-\frac{\varepsilon \sin \theta}{r}\left(\alpha \cos \theta r+(\alpha+\gamma) w+b \cos \theta r w+b w^{2}\right), \\
\dot{w} & =-\varepsilon(\gamma+b \cos \theta r+b w) w .
\end{aligned}
$$

Now taking as new independent variable the variable $\theta$ the previous differential system writes

$$
\begin{aligned}
\frac{d r}{d \theta} & =\varepsilon \cos \theta\left(\alpha \cos \theta r+(\alpha+\gamma) w+b \cos \theta r w+b w^{2}\right)+O\left(\varepsilon^{2}\right) \\
& =\varepsilon F_{1}(\theta, r, w)+O\left(\varepsilon^{2}\right), \\
\frac{d w}{d \theta} & =-\varepsilon(\gamma+b \cos \theta r+b w) w+O\left(\varepsilon^{2}\right) \\
& =\varepsilon F_{2}(\theta, r, w)+O\left(\varepsilon^{2}\right) .
\end{aligned}
$$

We shall apply the averaging theory described in Theorem 1 to the differential system (11). Using the notation introduced in Theorem 1 we have $t=\theta, T=2 \pi, \mathbf{x}=(r, w)^{T}$ and

$$
F(\theta, r, w)=\left(\begin{array}{c}
F_{1}(\theta, r, w) \\
F_{2}(\theta, r, w)
\end{array}\right), \quad \text { and } \quad f(r, w)=\left(\begin{array}{c}
f_{1}(r, w) \\
f_{2}(r, w)
\end{array}\right) .
$$

It is easy to check that system (9) satisfies all the assumptions of Theorem 1.

Now we compute the integrals (5), i.e.

$$
\begin{aligned}
& f_{1}(r, w)=\frac{1}{2 \pi} \int_{0}^{2 \pi} F_{1}(\theta, r, w) d \theta=\frac{1}{2} r(\alpha+b w), \\
& f_{2}(r, w)=\frac{1}{2 \pi} \int_{0}^{2 \pi} F_{2}(\theta, r, w) d \theta=-(\gamma+b w) w .
\end{aligned}
$$


The system $f_{1}(r, w)=f_{2}(r, w)=0$ has two solution $(0,0)$ and $(0,-\gamma / b)$. The first corresponds to an equilibrium point of system (11), but the second according Theorem 1 provides a periodic solution of system (11) because the Jacobian (6) takes the value $(\alpha-\gamma) \gamma / 2$ which is not zero by assumptions. Moreover the eigenvalues of the Jacobian matrix are $(\alpha-\gamma) / 2$ and $\gamma$.

Theorem 1 guarantees for $\varepsilon>0$ sufficiently small the existence of a periodic solution $(r(\theta, \varepsilon), w(\theta, \varepsilon))$ of system (11) such that $(r(0, \varepsilon), w(0, \varepsilon))=(0,-\gamma / b)+O(\varepsilon)$. Hence, system (10) has a periodic solution $(r(t, \varepsilon), \theta(t, \varepsilon), w(t, \varepsilon))$ such that $(r(0, \varepsilon), \theta(0, \varepsilon), w(0, \varepsilon))=(0,0,-\gamma / b)+O(\varepsilon)$. Consequently, system (9) has the periodic solution $(u(t, \varepsilon), v(t, \varepsilon), w(t, \varepsilon))$ such that $(u(0, \varepsilon), v(0, \varepsilon), w(0, \varepsilon))=$ $(0,0,-\gamma / b)+O(\varepsilon)$. Then, system (8) has the periodic solution $(X(t, \varepsilon), Y(t, \varepsilon), Z(t, \varepsilon))$ such that $(X(0, \varepsilon), Y(0, \varepsilon), Z(0, \varepsilon))=(0, \gamma / b, \gamma / b)+O(\varepsilon)$. Finally, for $\varepsilon>0$ sufficiently small system (7) has a periodic solution $(x(t, \varepsilon), y(t, \varepsilon), z(t, \varepsilon))$ such that $(x(0, \varepsilon), y(0, \varepsilon), z(0, \varepsilon))=(0, \varepsilon \gamma / b, \varepsilon \gamma / b)+O\left(\varepsilon^{2}\right)$. Therefore, it is a periodic solution starting at the equilibrium point located at the origin of coordinates when $\varepsilon=0$. This completes the proof of the theorem.

\section{Local dynamics at equilibria}

The eigenvalues at the equilibrium $O$ are real when $a^{2}-4 \geq 0$, namely $-c$ and $a / 2 \pm \sqrt{a^{2}-4} / 2$. Hence, $O$ is asymptotically stable whenever $c>0$ and $a \leq-2$, and unstable on $c<0$ or $a \geq 2$. On $a^{2}-4<0$ the eigenvalues are complex and $O$ is stable whenever $c>0$ and $-2<a \leq 0$ and unstable on $c<0$ or $0<a \leq 2$.

When $c \neq 0$ a Hopf bifurcation may occur at $O$ when $a=0$ because the eigenvalues are $-c$ and $\pm i$. Indeed, the first condition of Hopf bifurcation is fulfilled, $\left.\operatorname{Re}(d \lambda / d a)\right|_{a=0, \lambda=i}=1 / 2 \neq 0$. We need further to determine the first Lyapunov coefficient. To this end, write system (2) at $a=0$ in the form

$$
\dot{X}=J_{0} X+F(X)
$$

where $X=\left(\begin{array}{ll}x y z & z\end{array}\right)^{T}, J_{0}=J(0, c)$ and $F(X)=\left(\begin{array}{llll}0 & 0 & b & y z\end{array}\right)^{T}$. Write further $F(X)=B(X, X) / 2$ where

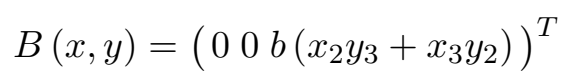

for any two vectors $x=\left(x_{1} x_{2} x_{3}\right)$ and $y=\left(y_{1} y_{2} y_{3}\right)$. A complex eigenvector $q$ corresponding to the eigenvalue $i, J_{0} q=i q$, respectively, an adjoint complex eigenvector $p, J_{0}^{T} p=-i p$, can be determined from the above eigenvectors $q_{+}$and $p_{+}$for $a=0$ and are given by

$$
q=\left(\begin{array}{l}
i \\
1 \\
0
\end{array}\right), \quad p=\left(\begin{array}{c}
\frac{1}{2} i \\
\frac{1}{2} \\
-\frac{i}{2 c-2 i}
\end{array}\right)
$$

which satisfy $\langle p, q\rangle=1$. Here we use $\langle u, v\rangle=\sum_{i=1}^{n} \bar{u}_{i} v_{i}$. With these notations, the first Lyapunov coefficient (see [Kuznetsov, 1995]) is $\ell_{1}(0)$ given by

$$
\frac{1}{2 \omega_{0}} \operatorname{Re}\left[-2\left\langle p, B\left(q, J_{0}^{-1} B(q, \bar{q})\right)\right\rangle+\left\langle p, B\left(\bar{q},\left(2 i \omega_{0} I_{3}-J_{0}\right)^{-1} B(q, q)\right)\right\rangle\right],
$$

which leads to $\ell_{1}(0)=0$. This means that the Hopf bifurcation is degenerate. Moreover, the second Lyapunov coefficient is also zero, $\ell_{2}(0)=0$, which implies that the degeneracy of the Hopf bifurcation does not give rise to a non-degenerate Bautin bifurcation. Hence, no conclusion using this analysis can be drawn on the existence of periodic orbits in the system (2) bifurcating from 0 when $a=0$ and $c \neq 0$. The second paper's result is the following theorem.

Theorem 3. Let $c=a<0$ and $b \neq 0$, then the Rössler-type system (2) has a Hopf bifurcation at the equilibrium point $(-a c / b, c / b,-c / b)$, and a stable periodic orbit borns at this equilibrium for $a-c>0$ sufficiently small, which in the normalized system to $(2)$ is near a circle of radius $\sqrt{\frac{1}{2\left(a^{2}+1\right)}(a-c)}$. 
Proof. Consider in the following system (2) in a neighborhood of the equilibrium point $A(-a c / b, c / b,-c / b)$ of course with $b \neq 0$. Translating $A$ to the origin by $x \rightarrow x+a c / b, y \rightarrow y-c / b$ and $z \rightarrow z+c / b$, system (2) becomes

$$
\dot{x}=-y-z, \quad \dot{y}=x+a y, \quad \dot{z}=-c y+b y z .
$$

The characteristic polynomial of system (13) at $O$ is $P(\lambda)=\lambda^{3}-a \lambda^{2}+\lambda-c$, which has the roots $a$ and $\pm i$ if and only if $c=a$. Since

$$
\left.\operatorname{Re} \frac{d \lambda}{d c}\right|_{c=a, \lambda=i}=\frac{-1}{2\left(a^{2}+1\right)} \neq 0,
$$

a potential Hopf bifurcation arises when $a c>0$ at $c=a$.

$$
\text { At } c=a \text { we have } J_{a}=\left(\begin{array}{ccc}
0 & -1 & -1 \\
1 & a & 0 \\
0-a & 0
\end{array}\right), q=\frac{1}{\sqrt{2}(a-i)}\left(\begin{array}{c}
i a+1 \\
-i \\
a
\end{array}\right) \text { and } p=\frac{1}{\sqrt{2}}\left(\begin{array}{l}
i \\
1 \\
1
\end{array}\right) \text {, where } J_{a} q=i q
$$

and $J_{a}^{T} p=-i p$, respectively.

Since an eigenvalue of $J_{a}$ is $\lambda_{1}=a$, a Hopf bifurcation may occur only when $a<0$. Proceeding as above, we find that $B(x, y)$ has the same form as in $(12)$ which leads to

$$
\ell_{1}(0)=-\frac{\sqrt{2}}{12} \frac{a^{2} b^{2}}{\left(a^{2}+1\right)\left(a^{2}+4\right)} .
$$

Since $\ell_{1}(0) \neq 0$ system (2) undergoes a Hopf bifurcation for all $b \neq 0$ and $a<0$ at the equilibrium $A$. A periodic solution bifurcates from the equilibrium $A$ when $c$ crosses $a$ with $a<0$ and $|c-a|$ small enough. More exactly, because $\ell_{1}(0)<0$ and $\left.\operatorname{Re}(d \lambda / d c)\right|_{c=a, \lambda=i}<0$, a unique and stable periodic solution exists for $c<a$ and $|c-a|$ is sufficiently small. In addition, the equilibrium $A$ is unstable on $c<a$ and stable on $c>a$. The periodic solution in the normalized system to $(2)$ is near a circle of radius $r \simeq \sqrt{-\frac{1}{2\left(a^{2}+1\right)}(c-a)}$ when $c-a<0$ is sufficiently small.

From Theorem 3 the local system's dynamics around the equilibrium $A$ in terms of periodic orbits is well characterized for all $a<0$ and $|c-a|$ small enough. This is not the case for the equilibrium $O$ for the values of the parameters $(a, c)$ around $(0, c)$. When $c \neq 0$ and $a=0$ the two equilibria $O$ and $A$ are still different but $\ell_{1}(0)=0$, so the Hopf bifurcation does not exist anymore, while for $a=c=0$ a degenerate fold-Hopf bifurcation occurs studied in Theorem 2.

Remark 3.1. Notice that we have proved that a Hopf bifurcation occurs at $c=a \leq 0$. In fact, at $c=a=0$ we have a fold-Hopf bifurcation. We also note that for $c=a<0$ if one chooses other eigenvectors, for example $q=\left(\frac{1}{a}(i a+1)-\frac{i}{a} 1\right)^{T}$ and $p=\frac{a}{2(a+i)}(i 11)^{T}$, we find

$$
\ell_{1}(0)=\frac{b^{2}}{a\left(a^{2}+1\right)\left(a^{2}+4\right)},
$$

which is different from (14) but has the same sign since $a<0$.

\section{Conclusions}

In this work we studied the behavior of a three-dimensional Rössler-type differential system in terms of the existence of periodic orbits emerging from degenerate fold-Hopf bifurcations. While the local behavior of a general differential system undergoing degenerate fold-Hopf bifurcations is not known in the literature, we showed that the averaging theory for detecting periodic orbits is a viable approach that can be applied at least for particular differential systems undergoing the bifurcation. Two open problems arise from this work related to the local behavior at the equilibria of system (2). More exactly, the system's behavior is not completely known when the parameters satisfy: 1) $a \neq 0$ and $c$ is small enough around 0 , respectively, 2) $c \neq 0$ and $a$ is small enough around 0 . 


\section{Appendix}

In this section we provide the reader the main results on the non-degenerate fold-Hopf bifurcations. More details can be found in [Kuznetsov, 1995].

We write in this appendix $x=0$ and $\alpha=0$ for $x=(0,0,0)$ and $\alpha=(0,0)$. Expanding $f(x, \alpha)$ with respect to $x$ at $x=0$, the system (1) reads

$$
\dot{x}=a(\alpha)+J(\alpha) x+F(x, \alpha),
$$

where $a(0)=0$ and $F(x, \alpha)=O\left(\|x\|^{2}\right)$. The system can be further put in the form

$$
\begin{aligned}
& \dot{u}=\Gamma(\alpha)+\nu(\alpha) u+g(u, z, \bar{z}, \alpha), \\
& \dot{z}=\Omega(\alpha)+\lambda(\alpha) z+h(u, z, \bar{z}, \alpha),
\end{aligned}
$$

where $u=\left\langle p_{0}(\alpha), x\right\rangle, z=\left\langle p_{1}(\alpha), x\right\rangle$ and $p_{0}(\alpha) \in \mathbb{R}^{3}, p_{1}(\alpha) \in \mathbb{C}^{3}$ are two adjoint eigenvectors given by

$$
J^{T}(\alpha) p_{0}(\alpha)=\nu(\alpha) p_{0}(\alpha) \text { and } J^{T}(\alpha) p_{1}(\alpha)=\bar{\lambda}(\alpha) p_{1}(\alpha)
$$

such that

$$
\left\langle p_{0}(\alpha), q_{0}(\alpha)\right\rangle=\left\langle p_{1}(\alpha), q_{1}(\alpha)\right\rangle=1 \text { and }\left\langle p_{1}(\alpha), q_{0}(\alpha)\right\rangle=\left\langle p_{0}(\alpha), q_{1}(\alpha)\right\rangle=0,
$$

for all $\|\alpha\|$ small enough. The vectors $q_{0}(\alpha), q_{1}(\alpha)$ are two eigenvectors corresponding to the eigenvalues $\nu(\alpha), \lambda=\mu(\alpha)+i \omega(\alpha)$, i.e. $J(\alpha) q_{0}(\alpha)=\nu(\alpha) q_{0}(\alpha)$ and $J(\alpha) q_{1}(\alpha)=\lambda(\alpha) q_{1}(\alpha)$. We can write

$$
x=u q_{0}(\alpha)+z q_{1}(\alpha)+\bar{z} \bar{q}_{1}(\alpha) .
$$

Here

$$
\Gamma(\alpha)=\left\langle p_{0}(\alpha), a(\alpha)\right\rangle \quad \text { and } \quad \Omega(\alpha)=\left\langle p_{1}(\alpha), a(\alpha)\right\rangle
$$

are smooth functions of $\alpha$ with $\Gamma(0)=\Omega(0)=0$ and

$$
\begin{aligned}
& g(u, z, \bar{z}, \alpha)=\left\langle p_{0}(\alpha), F\left(u q_{0}(\alpha)+z q_{1}(\alpha)+\bar{z} \bar{q}_{1}(\alpha), \alpha\right)\right\rangle, \\
& h(u, z, \bar{z}, \alpha)=\left\langle p_{1}(\alpha), F\left(u q_{0}(\alpha)+z q_{1}(\alpha)+\bar{z} \bar{q}_{1}(\alpha), \alpha\right)\right\rangle,
\end{aligned}
$$

are smooth functions of their variables whose Taylor expansions in $u, z, \bar{z}$ start with quadratic terms

$$
\begin{aligned}
& g(u, z, \bar{z}, \alpha)=\sum_{j+k+l \geq 2} \frac{1}{j ! k ! l !} g_{j k l}(\alpha) u^{j} z^{k} \bar{z}^{l}, \\
& h(u, z, \bar{z}, \alpha)=\sum_{j+k+l \geq 2} \frac{1}{j ! k ! l !} h_{j k l}(\alpha) u^{j} z^{k} \bar{z}^{l} .
\end{aligned}
$$

Using the changes

$$
\begin{aligned}
v= & u+\delta_{0}+\delta_{1} u+\delta_{2} z+\delta_{3} \bar{z}+\frac{1}{2} V_{020} z^{2}+\frac{1}{2} V_{002} \bar{z}^{2}+V_{110} u z+V_{101} u \bar{z}, \\
w= & z+\Delta_{0}+\Delta_{1} u+\Delta_{2} z+\Delta_{3} \bar{z}+\frac{1}{2} W_{200} u^{2}+\frac{1}{2} W_{020} z^{2}+\frac{1}{2} W_{002} \bar{z}^{2} \\
& +W_{101} u \bar{z}+W_{011} z \bar{z}
\end{aligned}
$$

where $\delta_{i}(\alpha), \Delta_{i}(\alpha)$ are smooth functions, $\delta_{i}(0)=\Delta_{i}(0)=0, i=0,1,2,3$, the system (16) can be further brought to Poincaré normal form. More exactly we have the following Theorem [Kuznetsov, 1995].

Theorem 4. If (G.1) $g_{200}(0) \neq 0$, then there exists a locally defined smooth, invertible variable transformation of the form (17), smoothly depending on the parameters, that for $\|\alpha\|$ small enough brings the system (16) in the form

$$
\begin{aligned}
\dot{v}= & \gamma(\alpha)+\frac{1}{2} G_{200}(\alpha) v^{2}+G_{011}(\alpha)|w|^{2}+\frac{1}{6} G_{300}(\alpha) v^{3} \\
& +G_{111}(\alpha) v|w|^{2}+O\left(\|(v, w, \bar{w})\|^{4}\right) \\
\dot{w}= & R(\alpha) w+H_{110}(\alpha) v w+\frac{1}{2} H_{210}(\alpha) v^{2} w+\frac{1}{2} H_{021}(\alpha) w|w|^{2} \\
& +O\left(\|(v, w, \bar{w})\|^{4}\right)
\end{aligned}
$$


where $v \in \mathbb{R}, w \in \mathbb{C}$ and $\gamma(\alpha), G_{j k l}(\alpha)$ are real-valued smooth functions while $R(\alpha), H_{j k l}(\alpha)$ are complexvalued smooth functions such that $\gamma(0)=0, R(0)=i \omega_{0}$. Their expressions at $\alpha=0$ are given in the Appendix.

If in addition (G.2) $G_{011}(0) \neq 0$, the Poincaré form (18) can be further reduced to (19) by means of time reparametrization

$$
d t=\left(1+e_{1}(\alpha) v+e_{2}(\alpha)|w|^{2}\right) d \tau
$$

and transformations

$$
u=v+e_{4}(\alpha) v+\frac{1}{2} e_{3}(\alpha) v^{2} \text { and } z=w+K(\alpha) v w
$$

where $e_{i} \in \mathbb{R}, K \in \mathbb{C}$ are smooth functions and $e_{4}(0)=0$. More exactly, the system (18) is locally smoothly orbitally equivalent near the origin to the system

$$
\begin{aligned}
& \dot{u}=\delta(\alpha)+B(\alpha) u^{2}+C(\alpha)|z|^{2}+O\left(\|u, z, \bar{z}\|^{4}\right) \\
& \dot{z}=\Sigma(\alpha) z+D(\alpha) u z+E(\alpha) u^{2} z+O\left(\|u, z, \bar{z}\|^{4}\right),
\end{aligned}
$$

where $u \in \mathbb{R}, z \in \mathbb{C}$ and $\delta(\alpha), B(\alpha), C(\alpha), E(\alpha)$ are real-valued smooth functions while $\Sigma(\alpha), D(\alpha)$ are complex-valued smooth functions (given below for $\alpha=0$ ) such that $\delta(0)=0, \Sigma(0)=i \omega_{0}$.

Finally, if $(G .3) E(0) \neq 0$ is satisfied, using the linear scaling $u=\frac{B(\alpha)}{E(\alpha)} \xi, z=\frac{B^{3}(\alpha)}{C(\alpha) E^{2}(\alpha)} \zeta$ and the time-reparametrization, $t=\frac{E(\alpha)}{B^{2}(\alpha)} \tau$, the system (19) leads to normal form

$$
\begin{aligned}
& \dot{\xi}=\beta_{1}(\alpha)+\xi^{2}+s|\zeta|^{2}+O\left(\|(\xi, \zeta, \bar{\zeta})\|^{4}\right), \\
& \dot{\zeta}=\left(\beta_{2}(\alpha)+i \omega_{1}(\alpha)\right) \zeta+\left(\theta(\alpha)+i \omega_{2}(\alpha)\right) \xi \zeta+\xi^{2} \zeta+O\left(\|(\xi, \zeta, \bar{\zeta})\|^{4}\right),
\end{aligned}
$$

where $s=\operatorname{sign}[B(0) C(0)]= \pm 1$ and

$$
\begin{aligned}
\beta_{1}(\alpha) & =\frac{E^{2}(\alpha)}{B^{3}(\alpha)} \delta(\alpha), \quad \beta_{2}(\alpha)=\frac{E(\alpha)}{B^{2}(\alpha)} \operatorname{Re}(\Sigma(\alpha)), \\
\theta(\alpha)+i \omega_{2}(\alpha) & =\frac{D(\alpha)}{B(\alpha)}, \quad \omega_{1}(\alpha)=\frac{E(\alpha)}{B^{2}(\alpha)} \operatorname{Im}(\Sigma(\alpha)),
\end{aligned}
$$

with $\|(\xi, \zeta, \bar{\zeta})\|^{4}=\left(\xi^{2}+|\zeta|^{2}\right)^{2}$.

This form (20), for (G.4) $\theta_{0}=\theta(0) \neq 0$ and (G.5) the map $\alpha \longmapsto \beta(\alpha)$ is regular at $\alpha=0$, leads to the truncated normal form

$$
\begin{aligned}
& \dot{\xi}=\beta_{1}+\xi^{2}+s r^{2}, \\
& \dot{r}=r\left(\beta_{2}+\theta(\alpha) \xi+\xi^{2}\right), \\
& \dot{\varphi}=\omega_{1}+\omega_{2} \xi .
\end{aligned}
$$

The coefficients needed above in Theorem 4 are

$$
\begin{aligned}
& G_{200}(0)=g_{200}(0), G_{011}(0)=g_{011}(0), H_{110}(0)=h_{110}(0), \\
& G_{300}(0)=g_{300}(0)-\frac{6}{\omega_{0}} \operatorname{Im}\left(g_{110}(0) h_{200}(0)\right), \\
& G_{111}(0)=g_{111}(0)-\frac{1}{\omega_{0}}\left[2 \operatorname{Im}\left(g_{110}(0) h_{011}(0)\right)+\operatorname{Im}\left(g_{020}(0) h_{101}(0)\right)\right], \\
& H_{210}(0)=h_{210}(0)+\frac{i}{\omega_{0}}\left[h_{200}(0)\left(h_{020}(0)-2 g_{110}(0)\right)-\left|h_{101}(0)\right|^{2}-h_{011}(0) \bar{h}_{200}(0)\right], \\
& H_{021}(0)=h_{021}(0)+\frac{i}{\omega_{0}}\left(h_{011}(0) h_{020}(0)-\frac{1}{2} g_{020}(0) h_{101}(0)-2\left|h_{011}(0)\right|^{2}-\frac{1}{3}\left|h_{002}(0)\right|^{2}\right),
\end{aligned}
$$


respectively,

$$
\begin{aligned}
& B(0)=\frac{1}{2} G_{200}(0), C(0)=G_{011}(0), \\
& D(0)=H_{110}(0)-i \omega_{0} \frac{G_{300}(0)}{3 G_{200}(0)}, \\
& E(0)=\frac{1}{2} \operatorname{Re}\left[H_{210}(0)+H_{110}(0)\left(\frac{\operatorname{Re}\left(H_{021}(0)\right)}{G_{011}(0)}-\frac{G_{300}(0)}{G_{200}(0)}+\frac{G_{111}(0)}{G_{011}(0)}\right)-\frac{H_{021}(0) G_{200}(0)}{2 G_{011}(0)}\right] .
\end{aligned}
$$

\section{Acknowledgments}

The research of the three authors was supported by FP7-PEOPLE-2012-IRSES-316338. The second author is also partially supported by a MINECO grant MTM2013-40998-P, an AGAUR grant number 2014SGR568, and the grant FP7-PEOPLE-2012-IRSES-318999.

\section{References}

Algaba, A., Freire, E. \& Gamero, E. [1998], "Hypernormal Form for the Hopf-Zero Bifurcation", International Journal of Bifurcation and Chaos 08(10), pp. 1857-1887.

Castellanos, V., Llibre, J. \& Quilantán, I. [2013] "Simultaneous periodic orbits bifurcating from two zeroHopf equilibria in a tritrophic food chain model", J. Applied Mathematics and Physics 1, pp. 31-38.

Euzébio, R.D., Llibre, J. \& Vidal, C. [2015] "Zero-Hopf bifurcation in the FitzHugh-Nagumo system", Mathematical Methods in the Applied Sciences 38, pp. 4289-4299.

Kuznetsov, Y.A. [1995] Elements of Applied Bifurcation Theory, (Springer-Verlag).

Llibre, J. [2014], "Periodic orbits in the zero-Hopf bifurcation of the Rössler system", Romanian Astron. J. 24, pp. 49-60.

Llibre, J. \& Pérez-Chavela, E. [2014] "Zero-Hopf bifurcation for a class of Lorenz-type systems", Discrete and Continuous Dynamical Systems, Series B 19, pp. 1731-1736.

Rössler, O.E. [1976], "An equation for continuous chaos", Physics Letters 57A, pp. 397-398.

Verhulst, F. [1991], Nonlinear Differential Equations and Dynamical Systems, (Universitext, Springer).

Wei, Z., Moroz, I., Wang, Z., Sprott, J. C. \& Kapitaniak, T. [2016], "Dynamics at Infinity, Degenerate Hopf and Zero-Hopf Bifurcation for Kingni-Jafari System with Hidden Attractors", International Journal of Bifurcation and Chaos 26, 1650125. 